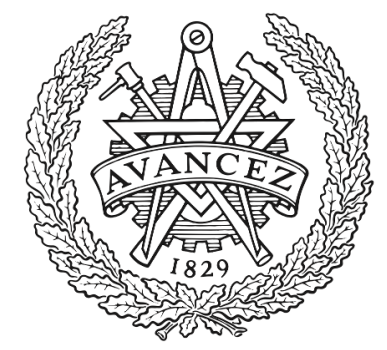

CHALMERS

UNIVERSITY OF TECHNOLOGY

\title{
First-principles study of the binding energy between nanostructures and its scaling with system size
}

Downloaded from: https://research.chalmers.se, 2023-04-26 05:16 UTC

Citation for the original published paper (version of record):

Tao, J., Jiao, Y., Mo, Y. et al (2018). First-principles study of the binding energy between nanostructures and its scaling with system size. Physical Review B, 97(15). http://dx.doi.org/10.1103/PhysRevB.97.155143

N.B. When citing this work, cite the original published paper. 


\title{
First-principles study of the binding energy between nanostructures and its scaling with system size
}

\author{
Jianmin Tao, ${ }^{1, *}$ Yang Jiao, ${ }^{2}$ Yuxiang Mo, ${ }^{1}$ Zeng-Hui Yang, ${ }^{3}$ Jian-Xin Zhu, ${ }^{4}$ Per Hyldgaard, ${ }^{2}$ and John P. Perdew ${ }^{1}$ \\ ${ }^{1}$ Department of Physics, Temple University, Philadelphia, Pennsylvania 19122-1801, USA \\ ${ }^{2}$ Department of Microtechnology and Nanoscience, MC2, Chalmers University of Technology, Sweden \\ ${ }^{3}$ Microsystem and Terahertz Research Center, China Academy of Engineering Physics, Chengdu, Sichuan 610200, China \\ ${ }^{4}$ Theoretical Division \& Center for Integrated Nanotechnologies, Los Alamos National Laboratory, Los Alamos, New Mexico 87545, USA
}

(Received 27 October 2017; revised manuscript received 6 February 2018; published 19 April 2018)

\begin{abstract}
The equilibrium van der Waals binding energy is an important factor in the design of materials and devices. However, it presents great computational challenges for materials built up from nanostructures. Here we investigate the binding-energy scaling behavior from first-principles calculations. We show that the equilibrium binding energy per atom between identical nanostructures can scale up or down with nanostructure size, but can be parametrized for large $\mathcal{N}$ with an analytical formula (in meV/atom), $E_{b} / \mathcal{N}=a+b / \mathcal{N}+c / \mathcal{N}^{2}+d / \mathcal{N}^{3}$, where $\mathcal{N}$ is the number of atoms in a nanostructure and $a, b, c$, and $d$ are fitting parameters, depending on the properties of a nanostructure. The formula is consistent with a finite large-size limit of binding energy per atom. We find that there are two competing factors in the determination of the binding energy: Nonadditivities of van der Waals coefficients and center-to-center distance between nanostructures. To decode the detail, the nonadditivity of the static multipole polarizability is investigated from an accurate spherical-shell model. We find that the higher-order multipole polarizability displays ultrastrong intrinsic nonadditivity, no matter if the dipole polarizability is additive or not.
\end{abstract}

DOI: 10.1103/PhysRevB.97.155143

\section{INTRODUCTION}

There is strong interest in nanomaterials, motivated by the development of nanotechnoloy and by their novel properties arising from quantum confinement. In particular, the discovery of various atomic-level materials has received overwhelming attention for their remarkable properties and wide-ranging applications [1]. A common feature of these materials is the strong adhesive van der Waals (vdW) force due to the instantaneous charge fluctuations. To understand the nature of the vdW force, a variety of experiments ranging from the smallest atomistic to the largest macroscopic scales have been performed recently [2-6]. However, details of many surprising phenomena due to the vdW interaction have not been well understood at the nanoscale [7].

Here we ask and answer another such question: Is there any binding-energy scaling formula showing the variation in binding energy with system size or number of atoms in a nanostructure?

The equilibrium binding energy between identical nanostructures is an important property involving microscopically the short-range contribution arising from the density overlap and the long-range vdW interaction. However, due to the large size of nanostructures, it presents great computational challenges. As such, an energy scaling formula showing the variation of equilibrium binding energy per atom with system size or number of atoms in a system is highly desired.

The equilibrium binding of nanostructures results from a competition between vdW attraction and the repulsion from the

\footnotetext{
*Corresponding author: jianmin.tao@temple.edu; http://www.sas.upenn.edu/ jianmint/
}

sum of kinetic and classical electrostatic energies. References [7-9] found unexpected long-range decays of the vdW interaction in certain systems. References $[10,11]$ found unexpected (strongly nonadditive) scaling of the vdW coefficient $C_{6}$ with system size in other systems. Reference [10] also found an unexpected scaling of the equilibrium binding energy of a $\mathrm{C}_{60}$ molecule to a multilayer graphene, as a function of the number of layers. The scaling of vdW coefficients affects the nanostructure binding but we must also establish the effects that enter via the size dependence of the repulsion.

To answer our scaling question, we study the size dependence of the equilibrium vdW binding energies (between identical nanostructures of various morphologies) using the consistent-exchange vdW-DF-cx version [12] of the vdW-DF method [13-15]. Like many other methods, the vdW-DF-cx functional is designed to produce good binding energy and equilibrium distance. Evidence that it does what it was designed to do, even for transition metals, is cited in Appendix A. To understand qualitatively the size dependence of the binding energy of nanostructures, we have to use another accurate model for the dynamic multipole polarizability to study the size dependence of the vdW coefficients. Reference [16] found that the equilibrium binding energies between fullerenes could be estimated by summing the vdW series

$$
E_{\mathrm{vdW}}=\sum_{k=3}^{\infty} C_{2 k} / r^{2 k}
$$

to very high order. We point out the competition in $C_{2 k} / r^{2 k}$ $(k=3,4,5)$ between the size dependence of the vdW coefficient $C_{2 k}$ and that of the relevant power $r^{2 k}$ of the center-tocenter distance $r$ (which at equilibrium is roughly the sum of 



FIG. 1. Binding-energy scaling behavior (in meV/atom) of fullerenes in fcc solids (a), and of pairs of other identical nanoparticles: C-PAHs and BN-PAHs in AA stacking dimers (b), C-NTs and BN-NTs in AA stacking solids (c), as well as carbon nanowires (C-NWs) and boron-nitride substitutes (BN-NWs) in AA stacking dimers (d), with system size. $\mathcal{N}$ on the vertical axis is the total number of $\mathrm{C} / \mathrm{B} / \mathrm{N}$ atoms in a nanostructure. The dotted curve (yellow for carbon and green for $\mathrm{BN}$ ) is the analytic modeling of Eq. (4).

the vdW radii of the two nanostructures). We will also show that strong nonadditivity is necessarily present in the $\mathrm{vdW}$ coefficients $C_{8}$ and $C_{10}$ (which contribute to the equilibrium binding energy), even when it is absent from $C_{6}$. Finally, we will present a comprehensive consistent-exchange vdW-DF-cx survey of nanostructure binding energies.

Figure 1 shows the energy scaling behavior for a variety of nanomaterials. Our energy scaling formula [Eq. (4)] is obtained by fitting to the vdW-DF-cx results while paying attention to the nature of the interactions in the asymptotic limits (for various classes of nanostructure problems). We find that the binding-energy scaling behavior is largely due to the competing size effects of the vdW coefficients and the sum of the vdW radii of nanostructures, the latter of which also depend upon the system size or number of atoms in a system. The radii reflect the exponential wall of kinetic-energy repulsion and play a dominant role in determining the equilibrium intermolecular distance. It is the $\mathrm{vdW}$ coefficients together with equilibrium intermolecular distance that determine the energy scaling behavior of nanostructures.

\section{COMPUTATIONAL METHODS}

The binding energy per atom is defined as $E_{b} / \mathcal{N}$, with $\mathcal{N}$ being the total number of $\mathrm{C} / \mathrm{B} / \mathrm{N}$ atoms in a nanostructure. For nanotubes, $\mathcal{N}$ is the number of atoms per unit length (in $\AA$ ). All our calculations of binding energies and distances $d_{c c}$ (center to center) and $d_{w w}$ (wall to wall) were performed with QUANTUM ESPRESSO [17], using the vdW-DF-cx [12] nonlocal density functional. We used ultrasoft pesudopotentials with a plane-wave energy cutoff at $680 \mathrm{eV}$. The binding energy $E_{b}$ is taken as the energy difference between relaxed nanostructures at infinite separation and at equilibrium, except for nanowires, in which the distance between two nanowires is fixed at $4.21 \AA$, the relaxed distance between carbon or boron-nitride nanowires (C-NWs or BN-NWs) with seven atoms, due to the high instability. For fullerenes, we used $2 \times 2 \times 2$ for the $k$ mesh. For polycyclic aromatic hydrocarbons (C-PAHs) and boron-nitride PAHs (BN-PAHS), only the $\Gamma$ point is included in the $k$ mesh, due to the large size of the cell. For nanotubes (where we only focus on armchair structure), 
TABLE I. Variation of binding energies per atom $E_{b} / \mathcal{N}$ (in meV/atom) of fullerenes in fcc solids, and of pairs of other identical nanoparticles: C-PAHs and BN-PAHs, C-NTs and BN-NTs, and carbon and BN nanowires with system size, and center-to-center $\left(d_{c c}\right)$ and wall-to-wall $\left(d_{w w}\right)$ distances $(\AA)$. We extrapolated experimental binding energies [37] at 850 to $0 \mathrm{~K}$ for fullerenes, except for $\mathrm{C}_{60}$, which was already extrapolated to $0 \mathrm{~K}$, by estimating the thermal correction per atom from the thermal heat capacity of $\mathrm{C}_{70}$ [37] and then making the same correction per atom from $C_{70}$ to the experimental values for fullerenes $C_{76}$ [38] and $C_{84}$ [37]. Experimental center-to-center distances are from Ref. [39]. All $d_{c c}$ between wires are fixed at $4.21 \AA$.

\begin{tabular}{|c|c|c|c|c|c|c|c|}
\hline & & $\mathcal{N}$ & $d_{c c}(\AA)$ & $d_{w w}(\AA)$ & $E_{b}^{\mathrm{cal}} / \mathcal{N}$ & $d_{c c}^{\operatorname{expt}}(\AA)$ & $E_{b}^{\text {expt }} / \mathcal{N}$ \\
\hline \multirow{4}{*}{ Fullerene } & $\mathrm{C}_{60}\left(I_{h}\right)$ & 60 & 9.96 & 3.05 & 35.0 & 10.02 & $28-35$ \\
\hline & $\mathrm{C}_{70}\left(D_{5 h}\right)$ & 70 & 10.52 & 2.99 & 32.9 & 10.61 & $26-32$ \\
\hline & $\mathrm{C}_{76}\left(D_{2}\right)$ & 76 & 10.92 & 2.92 & 32.2 & 10.94 & $28-29$ \\
\hline & $\mathrm{C}_{84}\left(D_{2}\right)$ & 84 & 11.06 & 2.78 & 31.6 & 11.36 & $29-35$ \\
\hline \multirow{5}{*}{ C-PAH } & Benzene & 6 & 4.07 & - & 17.4 & & \\
\hline & Naphthalene & 10 & 3.95 & - & 22.4 & & \\
\hline & Anthracene & 14 & 3.90 & - & 25.0 & & \\
\hline & 2,3-Benzanthracene & 18 & 3.86 & - & 26.8 & & \\
\hline & Pentacene & 22 & 3.84 & - & 27.8 & & \\
\hline \multirow{5}{*}{ BN-PAH } & $\mathrm{B}_{3} \mathrm{~N}_{3} \mathrm{H}_{6}$ & 6 & 3.98 & - & 21.8 & & \\
\hline & $\mathrm{B}_{5} \mathrm{~N}_{5} \mathrm{H}_{8}$ & 10 & 3.90 & - & 25.2 & & \\
\hline & $\mathrm{B}_{7} \mathrm{~N}_{7} \mathrm{H}_{10}$ & 14 & 3.85 & - & 26.8 & & \\
\hline & $\mathrm{B}_{9} \mathrm{~N}_{9} \mathrm{H}_{12}$ & 18 & 3.84 & - & 27.6 & & \\
\hline & $\mathrm{B}_{11} \mathrm{~N}_{11} \mathrm{H}_{14}$ & 22 & 3.83 & - & 28.4 & & \\
\hline \multirow{5}{*}{ C-NT } & $(3,3)$ & 5 & 7.28 & 3.08 & 44.3 & & \\
\hline & $(5,5)$ & 8 & 10.01 & 3.14 & 33.2 & & \\
\hline & $(10,10)$ & 16 & 16.78 & 3.15 & 23.6 & & \\
\hline & $(20,20)$ & 32 & 30.34 & 3.15 & 17.0 & & \\
\hline & $(40,40)$ & 64 & 57.47 & 3.15 & 11.9 & & \\
\hline \multirow{5}{*}{$\mathrm{BN}-\mathrm{NT}$} & $(3,3)$ & 5 & 7.18 & 2.95 & 43.3 & & \\
\hline & $(5,5)$ & 8 & 10.07 & 3.10 & 32.3 & & \\
\hline & $(10,10)$ & 16 & 16.97 & 3.11 & 22.9 & & \\
\hline & $(20,20)$ & 32 & 30.78 & 3.10 & 16.5 & & \\
\hline & $(40,40)$ & 64 & 58.38 & 3.10 & 11.8 & & \\
\hline \multirow{5}{*}{ Carbyne $\left(\mathrm{C}_{N}\right)$} & $\mathrm{C}_{8}$ & 8 & 4.21 & - & 31.4 & & \\
\hline & $\mathrm{C}_{10}$ & 10 & 4.21 & - & 27.7 & & \\
\hline & $\mathrm{C}_{12}$ & 12 & 4.21 & - & 25.6 & & \\
\hline & $\mathrm{C}_{14}$ & 14 & 4.21 & - & 24.4 & & \\
\hline & $\mathrm{C}_{16}$ & 16 & 4.21 & - & 23.8 & & \\
\hline \multirow{4}{*}{$\mathrm{BN}$-carbynes $(\mathrm{BN})_{N / 2}$} & $(\mathrm{BN})_{4}$ & 8 & 4.21 & - & 35.4 & & \\
\hline & $(\mathrm{BN})_{5}$ & 10 & 4.21 & - & 31.0 & & \\
\hline & $(\mathrm{BN})_{6}$ & 12 & 4.21 & - & 28.6 & & \\
\hline & $(\mathrm{BN})_{8}$ & 16 & 4.21 & - & 25.6 & & \\
\hline
\end{tabular}

we used $6 \times 6 \times 17$ to $1 \times 1 \times 17$ from $(3,3)$ to $(40,40)$. For nanowires, we used $3 \times 3 \times 2$ for all cases.

The nonlocal correlation part of the vdW-DF-cx is the same as in the original Rutgers-Chalmers [13] vdW-DF, which was derived from the fluctuation-dissipation theorem [18] for the description of the long-range vdW interaction [14]. The method contains both the leading-order and higher-order contributions, the latter of which are important for solids [16,19-22]. Its exchange part is based on a modified semilocal functional, which aims to improve the short-range description.

The vdW-DF-cx is a useful first-principles method, as supported by Appendixes A and B. The experimental geometries of fullerene solids (Appendix A) and their equilibrium binding energies (Table I and Appendix B) are well reproduced, although $C_{6}$ for a fullerene pair is not, as anticipated in Ref. [11]. Like SCAN+rVV10 [23], and with nearly the same binding energy curve, vdW-DF-cx predicts [24] a chemisorption minimum for graphene on $\mathrm{Ni}(111)$ at a distance of $2.1 \AA$ from the top nickel plane, in close agreement with experiment. Some of us have previously argued $[14,23]$ that a vdW functional can be accurate for equilibrium binding energies, even if it is not for asymptotic interactions.

To analyze the energy scaling behavior revealed from our calculation, we have to make use of the efficient yet accurate spherical-shell model within the single-frequency approximation (SFA) [25-27] to calculate the vdW coefficients between nanostructures, using the Casimir-Polder formula [28]

$$
C_{2 k}^{\mathrm{AB}}=\sum_{l_{1}=1}^{k-2} \frac{(2 k-2) !}{2 \pi\left[\left(2 l_{1}\right) !\left(2 l_{2}\right) !\right]} \int_{0}^{\infty} d u \alpha_{l_{1}}^{\mathrm{A}}(i u) \alpha_{l_{2}}^{\mathrm{B}}(i u),
$$

where $l_{2}=k-l_{1}-1$ with $k \geqslant 3$, and $l$ is the order of polarizability. In the SFA, we assume that (i) only valence electrons in the outermost subshell are polarizable, and (ii) the density 
is uniform inside the effective radius $R_{l}$ and zero otherwise. Within the SFA, the model dynamic multipole polarizability at imaginary frequency $\omega=i u$ takes the simple expression

$$
\alpha_{l}^{\mathrm{SFA}}(i u)=R_{l}^{2 l+1} \frac{\omega_{l}^{2}}{\omega_{l}^{2}+u^{2}} \frac{1-\rho_{l}}{1-\beta_{l} \rho_{l}},
$$

where $R_{l}$ is the effective outer radius of the shell, $\beta_{l}=$ $\omega_{l}^{2} \tilde{\omega}_{l}^{2} /\left[\left(\omega_{l}^{2}+u^{2}\right)\left(\tilde{\omega}_{l}^{2}+u^{2}\right)\right]$ describes the coupling of the sphere and cavity plasmon oscillations, and $\rho_{l}=(1-$ $\left.t_{l} / R_{l}\right)^{2 l+1}$ describes the shape of the shell, with $t_{l}$ being the shell thickness [26,29]. $\omega_{l}=\omega_{p} \sqrt{l /(2 l+1)}$ is the average sphere plasmon frequency, $\tilde{\omega}_{l}=\omega_{p} \sqrt{(l+1) /(2 l+1)}$ is the cavity plasmon frequency, and $\omega_{p}=\sqrt{4 \pi \bar{n}}$ is the average plasmon frequency of the extended electron gas, with $\bar{n}=N / V_{l}$ and $V_{l}$ being the $l$-dependent vdW volume. For fullerenes, $V_{l}=$ $(4 \pi / 3)\left[R_{l}^{3}-\left(R_{l}-t_{l}\right)^{3}\right]$. For nanotubes, we take a unit length to study. The volume per unit length is given as $V_{l}=2 \pi R_{0} t_{l}$, where $R_{0}$ is the average radius of a nanotube, which can be accurately calculated from first-principles methods [30-34], and $t_{l}$ is the effective thickness of the nanotube. Here $t_{l}=3.4$ bohrs, as adopted for fullerene molecules [26,29]. For BN-NT, we set $t_{l}=2.08$ bohrs [32]. For PAH and nanowire, we can make a similar analysis by taking carbon or $\mathrm{BN}$ atoms as a unit.

Since the geometry effect can be accounted for via $\alpha_{l}(0)$, the model is valid for any geometry [25]. Important many-body effects can enter the model via the input static dipole/multipole polarizability, which is taken from $a b$ initio calculations. Therefore, the model can achieve good universal accuracy. Because the model treats the molecule as a whole object, rather than decomposing the molecule as a collection of atoms as in atom pairwise models, it can take the nonadditivity effect on the static polarizability fully into account. Because of the earlier successes of our model, we assume that the frequency dependence is adequately modeled. Thus the error of the model can be size independent [25,27]. This is different from atom pairwise models, which often have growing error when system size increases.

\section{BINDING-ENERGY SCALING BEHAVIOR FOR NANOSTRUCTURES}

\section{A. Computational study}

Ball-to-ball interaction. Fullerene is an important class of nanomaterials with a variety of applications [35] in physics, chemistry, and materials science. The binding energy between fullerene pairs is a typical example of ball-ball interaction $[16,36]$. We have calculated the binding energies per atom of fullerene solids with the optimized fcc-type geometries (Appendix A). For $\mathrm{C}_{60}$, the experimental value was already extrapolated to $0 \mathrm{~K}$, while for others, the experimental values are available only at high temperature $(\sim 850 \mathrm{~K})$ [37], due to the strong vdW force. We have estimated the thermal energy correction ( $\sim 2 \mathrm{meV} /$ atom) for $\mathrm{C}_{70}$ solid, for which the heat capacity data are available [37]. For $C_{76}$ [38] and $\mathrm{C}_{84}$ [37], we take the same thermal correction per atom as for $\mathrm{C}_{70}$. From Table I we observe that the calculated binding energies are generally within the range of thermally corrected experiments, while the intermolecular distance $d_{c c}$ is between
TABLE II. Parameters $a, b, c$, and $d$ characterized by specific nanostructures that are determined by a fit to numerical binding energies in Table I.

\begin{tabular}{lcccc}
\hline \hline & $a$ & $b$ & $c$ & $d$ \\
\hline Fullerene pair & 28 & 28 & 11000 & 500000 \\
C-PAH pair & 30.6 & -52 & -220 & 300 \\
BN-PAH pair & 31 & -55 & -5 & 0 \\
C-NT pair & 8.0 & 250 & -350 & 100 \\
BN-NT pair & 7.8 & 250 & -150 & -1100 \\
C-NW pair & 16.7 & 111 & 30 & 100 \\
BN-NW pair & 16.3 & 150 & 20 & 0 \\
\hline \hline
\end{tabular}

the DFT-LSDA [16] (local spin-density approximation) and experimental value [39] (at room temperature). This suggests that the method used in this work is not only reliable for binding energy, but also for the center-to-center distance. See Appendix A for further detailed discussion.

Figure 1(a) shows that the binding energy of fullerenes per atom decreases slowly (only $3 \mathrm{meV} /$ atom from $\mathrm{C}_{60}$ to $\mathrm{C}_{84}$ ) with fullerene size, while Table II shows that the large-size limit of the binding energy is about $28 \mathrm{meV} /$ atom. From Table I we can see that the center-to-center distance $d_{c c}$ gradually becomes larger and larger from $\mathrm{C}_{60}$ to $\mathrm{C}_{84}$. With the increase of fullerene size, vdW coefficients per atom pair, in particular the higher-order ones (Table III), dramatically increase, while the sum of the vdW radii of fullerenes characterized by $d_{c c}$ also increases. These two factors have opposite effects on the energy scaling behavior, leading to the slow variation of the binding energy with system size. It is interesting to note that the wallto-wall distance $d_{w w}$ of fullerenes gets slightly shrunk from $\mathrm{C}_{60}$ to $\mathrm{C}_{84}$. This is because the vdW force between fullerenes increases from $\mathrm{C}_{60}$ to $\mathrm{C}_{84}$, pulling two fullerenes slightly closer. Note that in this context the large-size limit of fullerene is not graphene [40].

Plane-to-plane interaction. Polycyclic aromatic hydrocarbons (C-PAHs) are a large class of conjugated $\pi$-electron systems of great importance in many areas such as environmental chemistry, materials science, and astrochemistry [41]. The energy scaling behavior between C-PAHs reflects the plane-to-plane vdW interaction [42-44]. Here we focus on the binding energies per atom of C-PAH dimers with the optimized AA stacking. Crucial to this problem is the fact that the center-to-center distance $d_{c c}$ remains nearly the same for all C-PAHs, with a slight decreasing trend similar to $d_{w w}$ for fullerene pairs, as shown in Table I. This is because from benzene to pentacene, the vdW force increases, pulling two planar molecules slightly closer. Since the vdW coefficients per atom rapidly increase with system size [45], due in part to the nonadditivity arising from $\pi$-electron delocalization, while their center-to-center distance $d_{c c}$ does not change much, the binding energy between C-PAHs scales up rapidly, as shown in Fig. 1(b). A similar energy scaling behavior is also observed for boron-nitride (BN) substitutes [46] for the same reason, as shown in Table I and Fig. 1(b), respectively. From Table II we see that our energy scaling behavior predicts the same binding energy $30 \mathrm{meV} /$ atom between two identical long-chain limits of PAH and BN-PAH (AA stacking). Note that this limit is 
TABLE III. Scaling properties of the static multipole polarizabilities of fullerenes, C-NTs, and BN-NTs, with armchair $(m, m)$ and zigzag $(m, 0)$ structures, and the vdW coefficients between identical pairs. $\mathcal{N}$ is the number of atoms in a nanostructure. For NTs, it represents the number of atoms per unit length $(\AA)$. The static dipole polarizability and $C_{6}$ for fullerenes are the $a b$ initio values taken from Ref. [56], while the higher-order static polarizabilities and vdW coefficients are calculated in this work from Eqs. (2) and (3). For nanotubes, the static dipole polarizabilities are the $a b$ initio values taken from the literature [30-34], while all others are obtained in this work.

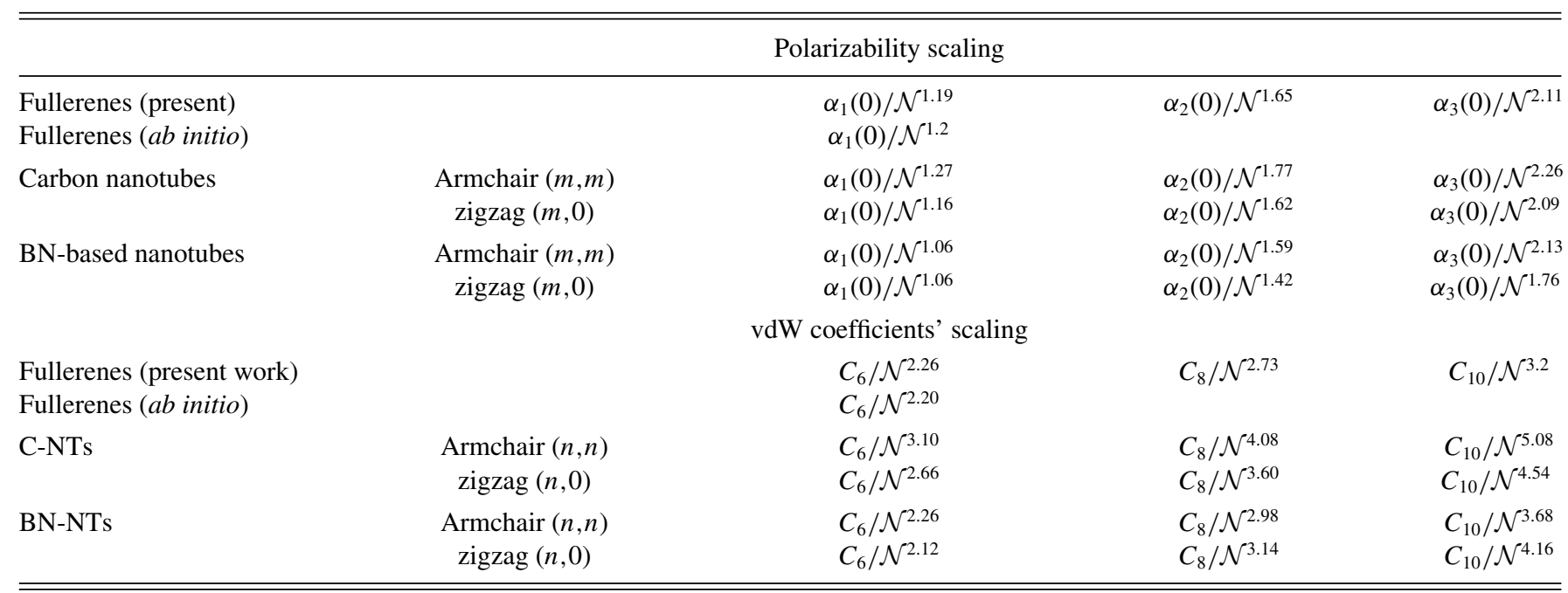

physically different from a bilayer of infinite two-dimensional sheets. The former are quasi-one-dimensional, while the latter are $2 \mathrm{D}$ materials.

Tube-to-tube interaction. Carbon nanotubes (C-NTs) are perhaps one of the most widely studied nanomaterials, due to their many unusual properties and applications [47]. Study of their energy scaling behavior is of broad interest. A C-NT has cylindrical symmetry. It is characterized by a pair of integer parameters $(n, m)$, with radius given by $(\sqrt{3} a / 2 \pi) \sqrt{n^{2}+m^{2}+n m}$, with $a$ being the bond length. When $n=m$, it takes the armchair structure, while for $n \neq m$, it takes the zigzag structure. Their size can be adjusted with $n$ or $m$. Here we focus on the binding energies per atom of the optimized close-packed solids of infinitely long armchair C-NTs. Figure 1(c) shows the variation of binding energy per atom with tube size for the AA stacking at the optimized geometry. From Fig. 1(c) we observe that when the size of C-NT increases from $(3,3)$ to $(40,40)$, the binding energy per atom drops significantly from 44.3 to $11.9 \mathrm{meV} /$ atom. Table I shows that the wall-to-wall distance $d_{w w}$ is nearly a constant with tube size, while the center-to-center distance $d_{c c}$ dramatically increases, a situation similar to fullerene. This largely decreases the vdW force, due to the fact that the increase of vdW coefficients with system size is weaker than the increase of the relevant power of the center-to-center distance $d_{c c}$ for C-NT pairs, leading to the decreasing trend of the binding energy with tube size. Clearly this trend has been followed by BN-NTs as shown in Fig. 1(c) and Table I. However, the binding energy for BN-NTs is slightly smaller than that for C-NTs. A possible explanation is that, because C-NTs and BN-NTs take structures similar to those of their bulks (the BN layered materials), the BN atoms in BN-NTs may not be all on the same surface, as they are for C-NTs. This will increase the band gap (5.5 eV) [48] of BN-NT (a situation similar to h-BN [49]) and thus decrease the vdW coefficients between BN-NTs, compared to C-NTs, as shown in Table III.
The energy scaling formula of Eq. (4) predicts the binding energies of $8.0 \mathrm{meV} /$ atom of C-NT, and $7.8 \mathrm{meV} /$ atom for BN-NT with AA stacking in the large size limit, which are slightly smaller than $11.5 \mathrm{meV} /$ atom for graphene (quantum Monte Carlo value) [40] and $9.9 \mathrm{meV} /$ atom of h-BN (MP2 value) [50] for AA stacking. Note that these comparisons only tell us that the large-size limits of C-NT and BN-NT should be around the binding energies of bilayer graphene and h-BN, because the large-size limits of C-NT and BN-NT are really not bilayer graphene and h-BN. The large-size binding energies are consistent with the wall-to-wall distances predicted by the vdW-DF-cx $(\sim 3.2 \AA$ for the large-size limit of C-NT and $\sim 3.1 \AA$ for BN-NT), which are smaller than the QMC value (3.5 $\AA$ for bilayer graphene) and MP2 value ( $3.3 \AA$ for $h-B N)$. This is because for bilayer graphene and h-BN, each atom of one sheet is close to atoms on the other sheet evenly, while in the large-size limits, a small fraction of the atoms on one tube is closer to atoms of the other tube, but a large fraction of the atoms on one tube will be far from any atoms of the other tube. These two competing factors seem to lead to a slight overall decrease of the binding energies in the large-size limits, compared to bilayer graphene and h-BN.

Wire-to-wire interaction. Carbyne is a carbon-based nanowire $(\mathrm{C}-\mathrm{NW})$ with an infinite chain of $s p$-hybridized carbon atoms, held together by either double or alternating single and triple atomic bonds. It displays unusual properties, such as strong chemical activity and extreme instability in ambient conditions. C-NW and its BN substitute (BN-NW) have attracted great attention recently [7,51-53], due to a variety of remarkable properties. Here we study the variation of binding energy per atom between two AA-stacked finite-length C-NWs with system size. Due to the instability of C-NW, the binding energy is calculated at a fixed distance between two C-NWs, rather than at the relaxed distance (see Computational Methods for detail). As shown by Fig. 1(d), the binding energy per atom between C-NWs decreases with system size. This is rather similar to those of fullerenes and nanotubes, but with 
much stronger size dependence. It is also opposite to the energy scaling behavior of PAHs. This feature has been inherited by its BN substitute. However, the binding energy between $\mathrm{BN}-\mathrm{NWs}$ is slightly stronger, due to the additional permanent dipole-dipole interaction between $\mathrm{B}$ and $\mathrm{N}$ atoms, a similar situation to BN-PAHs.

\section{B. Analytic fitting of binding-energy scaling formula}

Our binding-energy scaling formula should be useful in predicting the binding energy over a range of nanostructure sizes. Therefore, an analytic formula that fits to the binding energies of these nanostructures is highly desired. We find that the binding energies of these nanostructures can be fitted to the following analytic formula:

$$
E_{b} / \mathcal{N}=a+b / \mathcal{N}+c / \mathcal{N}^{2}+d / \mathcal{N}^{3},
$$

where $a, b, c$, and $d$ are the dimensionless fitting parameters given in Table II, and $\mathcal{N}$ is the number of atoms in a nanostructure. The energy is in unit of $\mathrm{meV} /$ atom. This formula reflects the nature of the large-size limits for the set of nanostructures.

From the binding energy scaling formula, we can predict binding energy or stability of nanostructures over a range of sizes. The form of Eq. (4) is that of a truncated expansion in $1 / \mathcal{N}$, and is not expected to be valid at small $\mathcal{N}$. However, we have also found a more complicated formula, with a better small- $\mathcal{N}$ behavior, that provides a similar fit to our calculated values.

\section{DISCUSSION}

To understand the energy scaling behavior of nanostructures, knowledge of the vdW coefficients is essential. Due to the direct relevance of the vdW coefficients to the static multipole polarizability, and in view of the relatively large size of the nanostructures, our starting point is the classical conducting solid or hollow sphere model, which is exact for slowly varying densities and thus very suitable for the study of nanostructures. In this model the static multipole polarizability satisfies [11]

$$
\alpha_{l}(0)=\left[\alpha_{1}(0)\right]^{(2 l+1) / 3},
$$

where $l=1$ (dipole), 2 (quadrupole), 3 (octupole), etc. (The dipole polarizability of a fullerene can be estimated [29] from $\alpha_{1}(0)=\left[R_{\mathcal{N}}+t / 2\right]^{3}$, where $R_{\mathcal{N}}$ is the average radius of the nuclear framework of a fullerene, and $t$ is the effective thickness of the shell.) Suppose the sphere contains $\mathcal{N}_{i}$ identical atoms with the static multipole polarizability $\alpha_{l}^{i}(0)$. Now let the volume of the sphere increase from $V_{i}$ to $V_{f}$ with fixed electron density, so that $\mathcal{N}_{i}$ will increase to $\mathcal{N}_{f}$. We seek interpolation relating the multipole polarizabilities at $\mathcal{N}_{i}$ and $\mathcal{N}_{f}$, the endpoints of the range over which we know $\alpha_{l}(0)$. The dipole polarizability per atom at $\mathcal{N}_{i}$ can be written as $\alpha_{1}^{i}(0) / \mathcal{N}_{i}=\alpha_{1}^{f}(0) / \mathcal{N}_{f}^{\left(1+\delta_{1}\right)}$. If $\delta_{1}=0$, the dipole polarizability is additive. Otherwise, it is nonadditive [54]. Similarly, the higher-order polarizabilities can be written as

$$
\alpha_{l}^{i}(0) / \mathcal{N}_{i}=\alpha_{l}^{f}(0) / \mathcal{N}_{f}^{\left(1+\delta_{l}\right)},
$$

where $\delta_{l}$ is a measure of nonadditivity of the multipole polarizability. Substituting Eq. (5) into Eq. (6) and performing some simple algebra, we can express the nonadditivity of the multipole polarizability in terms of that of the dipole polarizability as

$$
\delta_{l}=\left[(2 l+1)\left(1+\delta_{1}\right)-3\right] / 3-\frac{1}{3}[(2 l+1)-3]\left(\frac{\ln \mathcal{N}_{i}}{\ln \mathcal{N}_{f}}\right) .
$$

The last term of Eq. (7) is the size correction to the nonadditivity of the higher-order multipole polarizability. It vanishes for $l=1$ (dipole), and in the classical limit $\left(\mathcal{N}_{f} \rightarrow \infty\right)$. When the dipole polarizability is additive (i.e., $\delta_{1}=0$ ), we can still observe the strong nonadditivity of the higher-order multipole polarizability (i.e., $\delta_{l}>0$ ). Therefore, the nonadditivity of the higher-order multipole polarizability is an intrinsic property of a material. For any $\mathcal{N}_{i}<\mathcal{N}<\mathcal{N}_{f}$, we just replace $\mathcal{N}_{f}$ by $\mathcal{N}$ in Eq. (7). For the smallest possible data set $\mathcal{N}_{i}=$ $\mathcal{N}_{f}$, our formulas would predict $\delta_{l}=0$. In other words, the multipole polarizability at a single point $\mathcal{N}$ cannot deliver the physical nonadditivity. In order to identify the physical nonadditivity in which the initial value should be taken from an atom [26], we need the multipole polarizability at $\mathcal{N}$ or $\mathcal{N}_{f}$ that is reasonably larger than $\mathcal{N}_{i}$. The scaling properties for the static multipole polarizabilities of various nanostructures are given by Table III. [The dipole polarizabilities [55] of nanotubes are taken from Refs. [30-34], while the higher-order polarizabilities are estimated from Eq. (5).]

With the scaling properties of the static multipole polarizability, we can study the nonadditivity of the vdW coefficients. The vdW coefficients between two identical solid spheres, each having $\mathcal{N}$ atoms, take the simple form [26] $C_{6}=\alpha_{1}(0) \alpha_{1}(0) h_{6}(\bar{n}), C_{8}=\alpha_{1}(0) \alpha_{2}(0) h_{8}(\bar{n})$, and $C_{10}=$ $\alpha_{1}(0) \alpha_{3}(0) h_{10,1}(\bar{n})+\alpha_{2}(0) \alpha_{2}(0) h_{10,2}(\bar{n})$. Here $\bar{n}$ is the average valence electron density of the sphere, and $h_{6}-h_{10}$ are functions of $\bar{n}$ determined by the Casimir-Polder formula. Our calculation shows that $\bar{n}$ is nearly a constant with system size, so that the nonadditivity of vdW coefficients is essentially determined by the nonadditivity of the static multipole polarizability. According to Eqs. (5)-(7), we can express the nonadditivity of the vdW coefficients as

$$
C_{6}^{i} / \mathcal{N}_{i}^{2}=C_{6}^{f} / \mathcal{N}_{f}^{2+2 \delta_{1}} .
$$

If $\delta_{1}=0$ or the dipole polarizability is additive, so is $C_{6}$. Similarly, we have

$$
C_{8}^{i} / \mathcal{N}_{i}^{2}=C_{8}^{f} / \mathcal{N}_{f}^{2+\delta_{1}+\delta_{2}}, \quad C_{10}^{i} / \mathcal{N}_{i}^{2}=C_{10}^{f} / \mathcal{N}_{f}^{2+\delta_{1}+\delta_{3}},
$$

where $\delta_{l}$ are given by Eq. (7). (Note that $\delta_{1}+\delta_{3}=2 \delta_{2}$.) From Eqs. (7)-(9) we can see that the nonadditivity of the vdW coefficients $\left(2 \delta_{1}\right.$ for $C_{6}, \delta_{1}+\delta_{2}$ for $C_{8}$, and $\delta_{1}+\delta_{3}$ for $\left.C_{10}\right)$ largely arises from that of the multipole polarizability. If $C_{6}$ is additive, $C_{8}$ and $C_{10}$ are still nonadditive, because, even if $\delta_{1}=0, \delta_{2}$ and $\delta_{3}$ are not zero. This finding suggests that the nonadditivity of higher-order vdW coefficients essentially originates from the intrinsic nonadditivity of the multipole polarizability. The scaling properties for the vdW coefficients between nanostructures are also listed in Table III.

With knowledge of the nonadditivity of vdW coefficients, we can now explain the energy scaling behavior for fullerenes as follows. Let us consider the interaction between two identical classical solid spheres that are close enough [57]. Each 
sphere has a radius of $R=\left[\alpha_{1}(0)\right]^{1 / 3}$. The vdW coefficients between fullerenes are

$$
\begin{gathered}
C_{6} \sim \alpha_{1}(0) \alpha_{1}(0) \sim R^{6} \sim \mathcal{N}^{2\left(1+\delta_{1}\right)}, \\
C_{8} \sim \alpha_{1}(0) \alpha_{2}(0) \sim R^{8} \sim \mathcal{N}^{8\left(1+\delta_{1}\right) / 3}, \\
C_{10} \sim \alpha_{1}(0) \alpha_{3}(0) \sim R^{10} \sim \mathcal{N}^{10\left(1+\delta_{1}\right) / 3},
\end{gathered}
$$

where we have used Eq. (5). Comparison of Eqs. (8) and (9) with Eqs. (10)-(12) immediately leads to

$$
C_{2 j} /(2 R)^{2 j} \sim R^{0} .
$$

This means that if the spheres are close to each other, all energy terms of the vdW series are independent of $R$ or system size. This nonadditivity cancellation is valid for both solid spheres and hollow spheres with a cavity or fullerene, because our analysis for solid spheres is also valid for hollow spheres or fullerene. However, it was found [57] that this series diverges when two identical classical conducting spheres touch, but this spurious divergence can be removed without changing the asymptotic series. Nevertheless, the binding energy per atom $\left(\left[C_{2 j} /(2 R)^{2 j}\right] / \mathcal{N}\right)$ is decreasing with $\mathcal{N}$, as shown by Fig. 1(a).

This argument suggests that $E_{b} / \mathcal{N}$ for fullerenes should behave like $b / \mathcal{N}$ at large $\mathcal{N}$, while our Fig. 1(a) suggests that $E_{b} / \mathcal{N}$ tends to a nonzero constant $a$. This is not really a contradiction. The very strong and increasing-with- $k \mathcal{N}$ dependencies of $C_{2 k}$ in Eqs. (8)-(10) really are canceled by similar dependencies in $r^{2 k}$, but the argument is not quantitative enough to guarantee a perfect cancellation in $E_{b}$.

The physics behind the energy scaling behavior is now clear. The behavior observed in Figs. 1(a)-1(d) is a consequence of a competition between the nonadditivities in the vdW coefficients and in the vdW radii or center-to-center distance, which are saturated to the bulk values. For ball-ball interactions, there is large cancellation between $C_{2 j}$ and $(2 R)^{2 j}$, leading to a rather slow variation of the binding energy per atom with system size. For plane-plane interactions, the nonadditivity of $\mathrm{vdW}$ coefficients is dominant, because the parallel distance $d_{c c}$ is nearly a constant, leading to significant increase in binding energy. For tube-tube interactions, the nonadditivity of vdW coefficients becomes relatively less important due to the much larger size of tubes, compared to that of fullerenes, leading to a faster variation of the binding energy per atom with system size than that between fullerenes. There is a difference between $\mathrm{BN}$ atoms in BN-PAH and BN-NT. The reason is that BN atoms of BN-PAH can form $\pi$-electron delocalization, as in C-PAH. However, as in bulk h-BN, BN atoms of BN-NT cannot, because both BN-NT and bulk h-BN have large gaps $[48,49]$. This difference in bonding explains why the binding energy of BN-PAHs is greater than that of C-PAHs, but the binding energy for BN-NTs is slightly smaller than that for C-NTs. From BN-NW to h-BN bulk material, we can see that the energy gap evolves from a small value (nanowire) to a larger value $5.5 \mathrm{eV}$ (BN-NT), to a even larger value $5.9 \mathrm{eV}$ (h-BN), suggesting the deformation of $\mathrm{B}$ atoms from the surface of $\mathrm{N}$ atoms and thus a change in energy scaling behavior from nanowires to nanotubes. Due to the $\pi$-electron delocalization, the difference in binding energy scaling between C-PAH and $\mathrm{BN}-\mathrm{PAH}$ is the same as that between $\mathrm{C}-\mathrm{NW}$ and $\mathrm{BN}-\mathrm{NW}$ :
BN-based PAHs and BN-based nanowires show faster energy variation with system size than $\mathrm{C}$-based counterparts. The binding energy per atom of the nanowire dimers may decrease with increasing length due to dilution of the effect of covalent bonding between $\mathrm{C}$ atoms at the ends of the dimer.

\section{CONCLUSION}

The binding energy determines the stability of nanostructures and is therefore very important in the study of nanostructures. However, it has presented computational challenges. In this work we have studied the binding energy scaling behavior, showing the variation of the binding energy of nanostructures with system size or number of atoms in a nanostructure from a first-principles vdW-DF-cx method. We find that there is a binding-energy scaling formula between identical nanostructures. From the formula, we can predict the binding energy for a range of structure sizes. We illustrate this finding with fullerenes, PAHs, nanotubes, and nanowires. Apart from fullerenes, we chose AA stacking in our study. vdW-DF-cx is an effective vdW method, which is accurate in the prediction of binding energy and equilibrium distance of nanostructures, but its vdW coefficients for nanostructures may not be accurate, as shown in Appendix B (Table VII). To understand qualitatively the energy-scaling behavior, we have studied the nonadditivity of the vdW coefficients using another accurate vdW model. We find that the energy scaling behavior is determined by two competing factors: The size dependencies of the $\mathrm{vdW}$ coefficients and the center-to-center or intermolecular distance. This leads us to conclude that the energy-scaling behavior in part originates from the nonadditivity of the static multipole polarizability of nanostructures.

\section{ACKNOWLEDGMENTS}

The authors thank M. R. Pederson and R. Car for valuable comments and suggestions, J. Yang, G. Tian, and H. Peng for useful discussions and technical help, and Hong Tang for useful comments. Y.M. acknowledges support from the NSF under Grant No. CHE 1640584. J.T. was supported by the DOE Office of Science, Basic Energy Sciences (BES), under Grant No. DE-SC0018194. J.T. was also supported by Temple start-up from John P. Perdew. Y.J. and P.H. acknowledge support by the Swedish Research Council (VR) and the Swedish Research Foundation (SSF) under Contract SE13-0016. Z.Y. and J.P.P. were supported by the NSF under Grant No. DMR-1607868. Z.Y. was also supported by Science Challenge Project No. TZ2016003 (China). J.X.Z. acknowledges the support by the Center for Integrated Nanotechnologies, a DOE BES user facility. Computational support was provided by the HPC at Temple University and DOE NERSC as well as by the Swedish National Infrastructure for Computing through allocations at HPC2N (Umeå) and C3SE (Gothenburg).

\section{APPENDIX A: VDW-DF-CX CHARACTERIZATION OF FULLERENES}

Here we will show that the vdW-DF-cx functional predicts the geometries of fullerene solids in agreement with what is known experimentally about them. Other evidence for the 
(a)

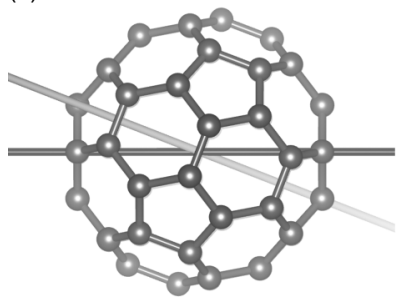

FIG. 2. The atomic configuration of $\mathrm{C}_{60}$ as seen perpendicular to (left panel) and along (right panel) the major symmetry axis, shown as a dark gray line in the left panel. The atomic configurations of $\mathrm{C}_{70}, \mathrm{C}_{76}$, and $\mathrm{C}_{80}$ fullerenes are shown as inserts in Fig. 1(a) of the main text. The major symmetry axis of $\mathrm{C}_{60}$ goes through a pair of hexagonal facets and it is experimentally found to be aligned with the [111] direction of the $\mathrm{C}_{60}$ fcc crystal, below $260 \mathrm{~K}$ [65]. The $\mathrm{C}_{60}$ molecule also has a secondary symmetry line (lighter gray line in both panels) which goes though a pair of pentagonal facets.

accuracy of vdW-DF-cx is presented in Appendix B and in Refs. [15,24,43,58-63].

Figure 2 shows the atom structure of $\mathrm{C}_{60}$ and the highsymmetry axis (dark gray line) going out of a pair of hexagonal facets; $\mathrm{C}_{60}$ also has a secondary axis (light gray line) going through a pair of pentagonal facets. The initial coordinates of $\mathrm{C}_{60}, \mathrm{C}_{70}, \mathrm{C}_{76}$, and $\mathrm{C}_{80}$ fullerenes are taken from the Supplemental Material of Ref. [64]. $\mathrm{C}_{60}$ and $\mathrm{C}_{70}$ have $I_{h}$ and $D_{5}$ symmetry, respectively. Since $\mathrm{C}_{76}$ and $\mathrm{C}_{80}$ have isomers, here we focus on $\mathrm{C}_{76}$ and $\mathrm{C}_{80}$ with $D_{2}$ symmetry.

Figure 3(a) shows the primitive (one molecule) and supercell (four molecules) geometries that we have used to model the fullerene crystals, as illustrated with $\mathrm{C}_{60}$. We assume that fullerenes are in crystal structures that are variations of fcc. Cohesive energies are extracted for (supercell) geometries that have been fully relaxed with the consistent-exchange vdW-DFcx version, using the BFGS quasi-Newton algorithm as available in variable-cell calculations (vc-relax) with the QUANTUM ESPRESSO package. We find no observable deformation of the individual fullerenes in any of the approximate-ground-state crystals structures that we have studied.

Table IV reports the details of fully unrestrained vdWDF-cx characterization of the optimal structure of fullerenes, when forced into a primitive cell [Fig. 3(a)]. Unconstrained relaxation was chosen, because we do not, except for the $\mathrm{C}_{60}$ crystal, have access to experimental information about alignment of the fullerene symmetry axis and of the fullerene crystal. This vdW-DF-cx characterization yielded the following identification of the nature of optimal structures: triclinic (rhombohedral) crystal symmetry for $\mathrm{C}_{60}$ and $\mathrm{C}_{84}$ (for $\mathrm{C}_{70}$ and $\mathrm{C}_{76}$ ). From these structures, we extracted the vdW-DF-cx

(a)
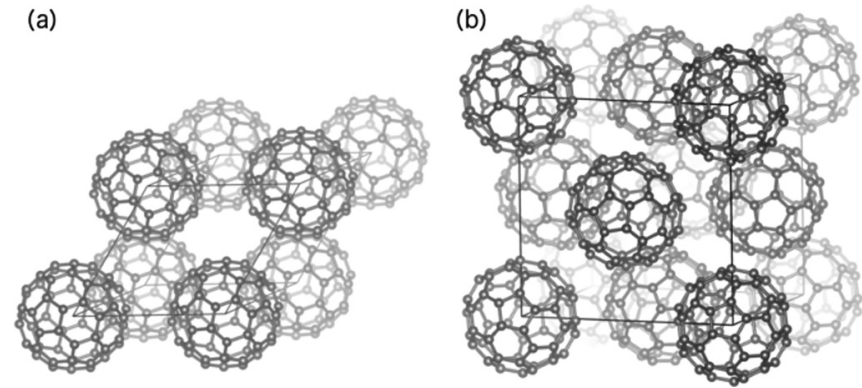

FIG. 3. The two types of unit cells used to model the ground state of fullerenes and thus extract vdW-DF-cx characterizations of sublimation energies $E_{b}$ of the molecular crystals. (a) The fundamental organization, which is fcc type. (b) An example of a 4- $\mathrm{C}_{60}$ supercell structure, denoted $F m \overline{3}$ mix for short, that represents an improved approximation for describing the ground state of $\mathrm{C}_{60}$ crystals.

results for the sublimation energies $E_{b}$ and for the wall-to-wall separations $d_{w w}$ (estimated as the distance to the nearest vertex or bond or facet on one molecule to the corresponding motif on the neighboring molecules). These results have been reported in the main text.

The $\mathrm{C}_{60}$ crystal motivates further theoretical characterizations because there is experimental data on structure [65]. We note that while our unconstrained relaxation (modeling a primitive cell) predicts a triclinic structure, the actual structure deviation is small (see Table IV). This difference is, in fact, expected. The ground state should have two different alignments of the symmetry axis relative to the crystal directions [65]. In choosing a primitive modeling, we are, on principle grounds, prevented from fully representing the actual $\mathrm{C}_{60}$ crystal ground state.

For a deeper discussion, we consider the role of the molecular orientation in the $\mathrm{C}_{60}$ crystal both in a primitive cell containing one molecule and in an improved modeling relying on 4 molecules per unit cell; the second modeling approach is illustrated in Fig. 3(b). The $\mathrm{C}_{60}$ crystal undergoes a phase transition at $260 \mathrm{~K}$. Above that temperature, all molecules can be considered equivalent, having free rotations, in a fcc primitive cell with one $\mathrm{C}_{60}$ molecule. Below this temperature, however, the $\mathrm{C}_{60}$ crystal is still fcc, but then in a supercell configuration of unknown size. There is no free rotation but a forced alignment of the major symmetry axis with the [111] direction of the fcc crystal cell. One can experimentally assign a rotational-angle value $\phi$ for molecules in the low-temperature systems [65]. However, the alignment must vary over the fcc-type supercell (of unknown size) which has a mixture of alignments: $15 \%$ molecules in a rotational configuration $R 38$ and about $85 \%$ in $R 98$.

TABLE IV. vdW-DF-cx results for primitive-cell lattice structures when permitting unconstrained unit-cell relaxations. They are all slightly distorted fcc as reflected in the lattice constants $(a, b, c)$ and unit-cell solid angles $(\alpha, \beta, \gamma)$.

\begin{tabular}{|c|c|c|c|c|}
\hline Molecule & Lattice system & $a / b / c(\AA)$ & $\alpha / \beta / \gamma(\mathrm{deg})$ & $V\left(\AA^{3}\right)$ \\
\hline $\mathrm{C}_{60}-I_{h}$ & Triclinic & $13.92 / 14.15 / 14.14$ & $91.2 / 90.8 / 90.9$ & 696 \\
\hline$C_{70}-D_{5 h}$ & Orthorhombic & $16.26 / 13.94 / 14.33$ & $90.0 / 90.0 / 90.0$ & 812 \\
\hline $\mathrm{C}_{76}-D_{2}$ & Orthorhombic & $16.95 / 15.36 / 13.91$ & $90.0 / 90.0 / 90.0$ & 905 \\
\hline $\mathrm{C}_{84}-D_{2}$ & Triclinic & $16.09 / 15.76 / 15.34$ & $90.3 / 88.4 / 90.0$ & 972 \\
\hline
\end{tabular}




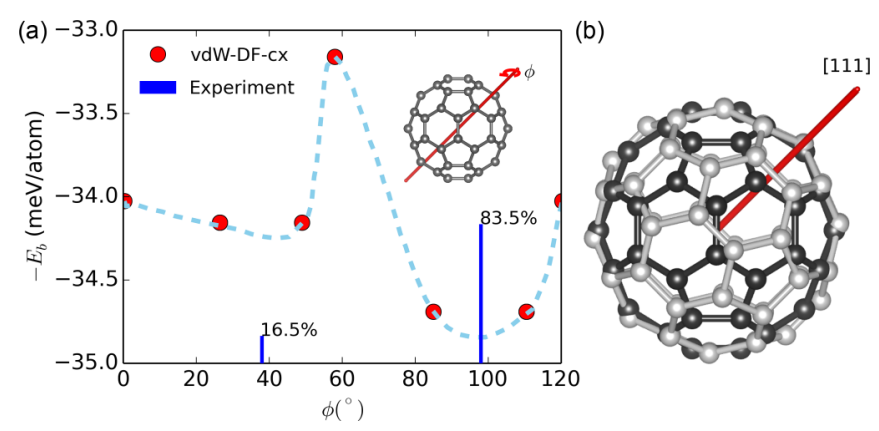

FIG. 4. Schematic of our test of vdW-DF-cx reliability in describing the internal $\mathrm{C}_{60}$ structural organization. These tests use a one-molecule cell, but track the role of different rotations $\phi$ of the $\mathrm{C}_{60}$ around the major symmetry axis. (a) vdW-DF-cx results for the energy variation of such states, denoted $R \phi$. The pair of vertical blue lines identifies the two experimentally observed, optimal rotational states that are both found in the ground state [65]. (b) Contrasts the atomic structure having such alignment of the [111] crystal axis with the major (black atoms and bonds) against that of alignment with the minor (gray atoms and bonds) symmetry axis.

Figure 4 summarizes the additional structure exploration that we have done to test the ability of vdW-DF-cx to characterize the structural motifs of the $\mathrm{C}_{60}$ ground state. The figure shows the sublimation-energy variation that results in a single-molecule unit-cell modeling as we vary the alignment of the major symmetry axis with the [111] crystal axis, all in an fcc structure; since the major axis has a threefold symmetry, it is only necessary to explore constrained relaxations in the range $0<\phi<120$. Tracking the relaxations in vdW-DF-cx, we thus identify a set of metastable configurations, red dots, with specific rotations but with a range of structural symmetries, as further described in Table V. This class of metastable fcc structures differs qualitatively from the previously mentioned $\mathrm{C}_{60}$ triclinic structure, which, instead, has an alignment with the secondary symmetry axis in $\mathrm{C}_{60}$. However, as we show later, the energy differences are very small.

The dashed line in the left panel of Fig. 4 represents a guide to the eye among those metastable configurations. We assume that a full exploration would stabilize major-axis configurations at more rotational values, when pursued at a supercell size that reflects the actual ground state. If we furthermore take the dashed line as an approximation for how such additional local minima would be distributed in energies, then we can expect optimal rotational values at around $\phi \approx 40^{\circ}$ and $\phi \approx 100^{\circ}$. It is heartening that these angles coincide with those that emerge as most prevalent in the mixture description obtained in the experimental characterization of $\mathrm{C}_{60}$, evident as vertical blue lines in Fig. 4.

Table $\mathrm{V}$ also reveals that structural optimization in the supercell indicates a very small preference for mixing different molecular rotations. Here again the relaxation is constrained to the experimentally observed major-axis alignment. In a supercell, however, we can allow individual molecules to relax to different orientation values. The structure identified as $F m \overline{3}$ mix is an example of an energetically favorable such metastable configuration. Like the actual but unknown ground-state supercell [65], this structure is characterized by having a mixture of molecular rotations.

Finally, Table VI lists the sublimation energies that arises when the [111] crystal axis (red line) is assumed to align with either the major or the secondary symmetry axis for the $\mathrm{C}_{60}$ crystal. For a single-molecule modeling, and among the cases with major-axis alignment, we find a best case, $F m \overline{3} R 111^{\circ}$, with a sublimation energy that lies with $0.1 \mathrm{meV} /$ atom of that of the triclinic structure (with the secondary-axis alignment). Also, although the energy differences are still very small, the $E_{b}$ ordering is reversed when instead we consider the best four-molecule supercell case (with correct alignment), denoted $F m \overline{3}$ mix.

Overall, we find that the vdW-DF-cx is able to reflect the known structural motifs of the $\mathrm{C}_{60}$ crystal (although the

TABLE V. Geometries and binding energies of metastable $\mathrm{C}_{60}$ crystals as optimized in vdW-DF-cx with constraints. Here, for the singlemolecule cell, we force the major $\mathrm{C}_{60}$ symmetry axis to be aligned with the crystal axis and identify metastable configurations, denoted $F m \overline{3} R \phi^{\circ}$ (red dots in Fig. 4), with specific rotation $\phi$ values. For the four-molecule conventional unit cell studies, we list all metastable configurations that we have found emerging from a fcc starting point while permitting all four rotational angles to differ. The low-energy structure $F m \overline{3} R 0^{\circ}$ has the same value for all four angles. The low-energy structure $F m \overline{3}$ mix is short for a four-molecule super cell in rotational configuration $R 9^{\circ} R 23^{\circ} R 37^{\circ} R 116^{\circ}$.

\begin{tabular}{|c|c|c|c|c|c|c|}
\hline Symmetry & Lattice system & $a / b / c(\AA)$ & $\alpha / \beta / \gamma(\operatorname{deg})$ & $V\left(\AA^{3}\right)$ & $d_{w w}(\AA)$ & $E_{b}(\mathrm{meV} /$ atom $)$ \\
\hline \multicolumn{7}{|c|}{ Experiment [65] } \\
\hline $0.835 F m \overline{3} R 98^{\circ}$ & Cubic & 14.04 & & 692 & & \\
\hline \multicolumn{7}{|c|}{ vdW-DF-cx; primitive cell } \\
\hline$F m \overline{3} R 0^{\circ}$ & Cubic & $14.10 / 14.10 / 14.10$ & $90.0 / 90.0 / 90.0$ & 701 & 3.06 & 34.0 \\
\hline$F m \overline{3} R 27^{\circ}$ & Rhombohedral & $14.08 / 14.08 / 14.08$ & $90.2 / 90.2 / 90.2$ & 698 & 3.11 & 34.1 \\
\hline$F m \overline{3} R 49^{\circ}$ & Rhombohedral & $14.08 / 14.08 / 14.08$ & $90.2 / 90.2 / 90.2$ & 698 & 3.11 & 34.1 \\
\hline$F m \overline{3} R 58^{\circ}$ & Rhombohedral & $14.14 / 14.14 / 14.14$ & $89.9 / 89.9 / 89.9$ & 707 & 3.09 & 33.2 \\
\hline$F m \overline{3} R 85^{\circ}$ & Rhombohedral & $14.07 / 14.07 / 14.07$ & $90.4 / 90.4 / 90.4$ & 697 & 3.11 & 34.7 \\
\hline$F m \overline{3} R 111^{\circ}$ & Rhombohedral & $14.07 / 14.07 / 14.07$ & $90.4 / 90.4 / 90.4$ & 697 & 3.11 & 34.7 \\
\hline \multicolumn{7}{|c|}{ vdW-DF-cx; 4 molecules/unit cell } \\
\hline$F m \overline{3} R 0^{\circ}$ & Cubic & $13.99 / 13.99 / 13.99$ & $90.0 / 90.0 / 90.0$ & 684 & 2.98 & 36.6 \\
\hline$F m \overline{3} \operatorname{mix}$ & Rhombohedral & $13.96 / 13.96 / 13.96$ & $90.7 / 90.7 / 90.7$ & 679 & 2.96 & 36.8 \\
\hline
\end{tabular}


TABLE VI. Comparison of geometries and binding energies of $\mathrm{C}_{60}$ crystals in metastable cubic/rhombohedral structures (in which the [111] crystal axis is kept aligned with major symmetry axis) and in alternative triclinic structures (in which [111] is found to be aligned with the secondary $\mathrm{C}_{60}$ symmetry axis). The former is a characteristics of the experimentally observed ground state, while the latter is what emerges in unconstrained relaxations in the one-molecule primitive cell.

\begin{tabular}{|c|c|c|c|c|c|c|}
\hline Symmetry & Lattice system & $a / b / c(\AA)$ & $\alpha / \beta / \gamma(\operatorname{deg})$ & $V\left(\AA^{3}\right)$ & $d_{w w}(\AA)$ & $E_{b}(\mathrm{meV} /$ atom $)$ \\
\hline \multicolumn{7}{|c|}{ Experiment [65] } \\
\hline $0.835 F m \overline{3} R 98^{\circ}$ & Cubic & 14.04 & & 692 & & \\
\hline \multicolumn{7}{|c|}{ vdW-DF-cx; primitive cell } \\
\hline- & Triclinic & $13.92 / 14.15 / 14.14$ & $91.2 / 90.8 / 90.9$ & 696 & 3.05 & 34.8 \\
\hline$F m \overline{3} R 0^{\circ}$ & Cubic & $14.10 / 14.10 / 14.10$ & $90.0 / 90.0 / 90.0$ & 701 & 3.06 & 34.0 \\
\hline$F m \overline{3} R 111^{\circ}$ & Rhombohedral & $14.07 / 14.07 / 14.07$ & $90.4 / 90.4 / 90.4$ & 697 & 3.11 & 34.7 \\
\hline \multicolumn{7}{|c|}{ vdW-DF-cx; 4 molecules/unit cell } \\
\hline- & Triclinic & $13.90 / 14.08 / 14.04$ & $91.2 / 91.0 / 90.8$ & 687 & 2.93 & 36.1 \\
\hline$F m \overline{3} \operatorname{mix}$ & Rhombohedral & $13.96 / 13.96 / 13.96$ & $90.7 / 90.7 / 90.7$ & 679 & 2.96 & 36.8 \\
\hline
\end{tabular}

$\mathrm{C}_{60}$ ground state is not fully known): (a) preference for a fcc-type supercell configuration with a mixture of rotational angles, (b) a preference for having predominantly a $\phi=100^{\circ}$ rotational state, and (c) a per-molecule volume value, which for the most favorable supercell representation, lies within $2 \%$ of the experimentally observed value $692 \AA^{3}$. We take this vdW-DF-cx progress as an indication that it can be used to predict the binding structures of the set of investigated nanostructured materials and that it is a good starting point for exploring energy scaling behavior.

\section{APPENDIX B: ASYMPTOTIC BINDING IN VDW-DF-CX}

Here we will show that vdW-DF-cx predicts equilibrium binding energies of fullerene solids in agreement with experiment, even though, as anticipated in Ref. [11], it is not accurate for the asymptotic interaction of a fullerene pair.

We have extracted the $\mathrm{C}_{6}$ values that correspond to an asymptotic vdW-DF-cx description, following prior discussions of the nature of the vdW-DF binding $[13,66]$. For the finite fullerene structures, this leads to a determination of the $\mathrm{C}_{6}$ coefficients using Eqs. (5)-(7) of Ref. [36]. The following information is presented to permit a discussion of differences in the asymptotic description from the hollow-shell model [56].

In the vdW-DF method, we work with a local-field susceptibility $\alpha$ and corresponding external-field susceptibility $\alpha_{\text {ext }}=\alpha /(1+4 \pi \alpha)$. This susceptibility (or plasmon propagator) depends on two spatial coordinates but can be represented in a gradient expansion. When investigating the asymptotic interactions, the relevant limit is $[13,66]$

$$
\alpha_{\text {ext,assym }}^{\mathrm{vdW}-\mathrm{DF}}(i u, r) \rightarrow \frac{n(r)}{u^{2}+\left[9 q_{0}(r)^{2} /(8 \pi)\right]^{2}} .
$$

This susceptibility limit is directly set by the inverse length scale $q_{0}$ that enters in the specification of the local plasmon dispersion $[13,14,66]$. We compute this susceptibility limit from the electron-density variation $n(\mathbf{r})$ that we have established in the underlying (full) vdW-DF-cx calculations.

From the approximation Eq. (B1) we determine, in turn, the asymptotic vdW-DF-cx description of nanostructure interaction from a Casimir-Polder expression of the molecular $C_{6}$ coefficients, using a numerical imaginary-frequency integration. The result is a description similar to Eq. (17) of Ref. [13]. We note that this $C_{6}$ limit is not an exhaustive representation of the full, regular vdW-DF-cx calculations, for reasons explained in Refs. [13,14,36,66].

Table VII reports our numerical extraction of such vdWDF-cx based $C_{6}$ coefficients for fullerenes with various number of carbon atoms $\mathcal{N}$, here contrasted with TDHF-based values cited in Table III of the main text. In general, the nonlocalcorrelation part of vdW-DF [13] (same as in vdW-DF-cx) leads to good $C_{6}$ values for small-to-medium sized molecules [67]. However, for the hollow fullerenes, the vdW-DF-cx values are about half the size of the results of the shell-model analysis based on TDHF calculations [56]. Also, the vdW-DF-cx results for $\mathrm{C}_{6} / \mathcal{N}^{2}$ is nearly independent of $\mathcal{N}$. Thus the vdW-DFcx descriptions does not reflect the $C_{6}$ nonadditivity that is expressed in the TDHF-based $C_{6}$ description [56] and hollowsphere model.

The vdW-DF-cx functional slightly overestimates the nanostructure binding energies $E_{b}$, but it is still useful for mapping the energy scaling as it is accurate on structure characterizations (Appendix A and Refs. [12,43,58,61]), as well as for nanostructure energy differences [12,24,59,61]. Table VII also reports a comparison of the vdW-DF-cx results for fullerene sublimation energies $E_{b}$ and raw experimental observations (no thermal correction); Table II reports the

TABLE VII. Asymptotic van der Waals interaction coefficients $C_{6}$ and molecular-crystal sublimation (or cohesion) energies $E_{b}$ for fullerenes. The van der Waals interaction coefficients are in atomic units (hartree for energy, bohr for distances) while $E_{b}$ is reported in $\mathrm{meV}$ per carbon atom. The vdW-DF-cx results for $E_{b}$ are listed for the primitive (one fullerene per) cell studies (see Appendix A). The experimental results $[37,38]$ are enthalpies of sublimation without thermal corrections.

\begin{tabular}{lcccccc}
\hline \hline & \multicolumn{2}{c}{ vdW-DF-cx } & \multicolumn{2}{c}{ TDHF [56] } & \multirow{2}{*}{ vdW-DF-cx } & Experiment \\
& $C_{6} / 10^{3}$ & $C_{6} / \mathcal{N}^{2}$ & $C_{6} / 10^{3}$ & $C_{6} / \mathcal{N}^{2}$ & $E_{b} / \mathcal{N}$ & $E_{b} / \mathcal{N}$ \\
\hline $\mathrm{C}_{60}$ & 55.2 & 15.33 & 100.1 & 27.80 & 35.0 & $25-32$ \\
$\mathrm{C}_{70}$ & 74.8 & 15.26 & 141.6 & 28.90 & 32.9 & $24-30$ \\
$\mathrm{C}_{76}$ & 88.1 & 15.25 & - & - & 32.2 & $26-27$ \\
$\mathrm{C}_{84}$ & 107.3 & 15.20 & 207.7 & 29.44 & 31.6 & $27-34$ \\
\hline \hline
\end{tabular}


comparison when the finite-temperature effect is removed from the experimental values.

For the C-PAH dimers in the sandwich or AA configuration, $\operatorname{CCSD}(\mathrm{T})$-based best estimates of the binding energy per atom for benzene, naphthalene, and anthracene [68] are 13.2, 17.8, and $21.1 \mathrm{meV}$ per carbon atom, versus the vdW-DF-cx values of $17.4,22.4$, and $25.0 \mathrm{meV}$ per carbon atom, respectively. For the corresponding benzene, naphthalene, and anthracene molecular crystals, the measured supplimation energies are 22, 41 , and $40 \mathrm{meV}$ per carbon atom, versus the vdW-DF-cx values of 25,46 , and $44 \mathrm{meV}$ per carbon atom [43]. Thus vdW-DF-cx captures the right trends with increasing particle size (being in these cases about $4 \mathrm{meV}$ per carbon atom higher than the best available estimates).

Moreover, vdW-DF-cx is accurate in first-principle characterizations of nanostructure-energy differences. It has proven useful for the description of both elastic deformations energies
$[12,15,60,62]$ and lattice vibrations [59-62]. For example, it can accurately reproduce the measurement of all libration modes in the naphthalene molecular crystal, characterizing the phonon dispersion to within $1 \mathrm{meV}$ (without any experimental input on the molecular-crystal structure) [62].

Some of us have previously (independently) discussed that it is possible for a density functional to fail in the asymptotic description but still be accurate at binding separations $[14,23]$. The vdW-DF-cx description of the fullerene crystals gives an example: Appendix A shows that vdWDF-cx does give an accurate description of structural motifs in fullerene crystals at binding separation even if $\mathrm{vdW}$ DF-cx is not accurate for (and does not give nonadditive) $C_{6}$ coefficients, Table VII. We also note that the vdW-DF$\mathrm{cx}$ is nonadditive in a different sense, namely in its description of the nonlocal correlation interaction at binding separations [69].
[1] Nanoclusters, Volume 1: A Bridge across Disciplines, edited by P. Jena and A. W. Castleman, Jr. (Elsevier, Amsterdam, 2010).

[2] G. Rance, D. Marsh, S. Bourne, T. Reade, and A. Khlobystov, van der Waals interactions between nanotubes and nanoparticles for controlled assembly of composite nanostructures, ACS Nano 4, 4920 (2010).

[3] P. Loskill, J. Puthoff, M. Wilkinson, K. Mecke, K. Jacobs, and K. Autumn, Macroscale adhesion of gecko setae reflects nanoscale differences in subsurface composition, J. R. Soc. Interface 10, 20120587 (2013).

[4] S. R. Na, J. W. Suk, R. S. Ruoff, R. Huang, K. M. Liechti, Ultra long-range interactions between large area graphene and silicon, ACS Nano 8, 11234 (2014).

[5] S. Tsoi, P. Dev, A. L. Friedman, R. Stine, J. T. Robinson, T. L. Reinecke, and P. E. Sheehan, van der Waals screening by singlelayer graphene and molybdenum disulfide, ACS Nano 8, 12410 (2014).

[6] S. Kawai, A. S. Foster, T. Björkman, S. Nowakowska, J. Björk, F. F. Canova, L. H. Gade, T. A. Jung, and E. Meyer, van der Waals interactions and the limits of isolated atom models at interfaces, Nat. Commun. 7, 11559 (2016).

[7] A. Ambrosetti, N. Ferri, R. A. DiStasio, Jr. and A. Tkatchenko, Wavelike charge density fluctuations and van der Waals interactions at the nanoscale, Science 351, 1171 (2016).

[8] J. F. Dobson, A. White, and A. Rubio, Asymptotics of the Dispersion Interaction: Analytic Benchmarks for van der Waals Energy Functionals, Phys. Rev. Lett. 96, 073201 (2006).

[9] S. Lebègue, J. Harl, T. Gould, J. G. Ángya, G. Kresse, and J. F. Dobson, Cohesive Properties and Asymptotics of the Dispersion Interaction in Graphite by the Random Phase Approximation, Phys. Rev. Lett. 105, 196401 (2010).

[10] V. V. Gobre and A. Tkatchenko, Scaling laws for van der Waals interactions in nanostructured materials, Nat. Commun. 4, 2341 (2013).

[11] A. Ruzsinszky, J. P. Perdew, J. Tao, G. I. Csonka, and J. M. Pitarke, van der Waals Coefficients for Nanostructures: Fullerenes Defy Conventional Wisdom, Phys. Rev. Lett. 109, 233203 (2012).

[12] K. Berland and P. Hyldgaard, Exchange functional that tests the robustness of the plasmon description of the van der Waals density functional, Phys. Rev. B 89, 035412 (2014).
[13] M. Dion, H. Rydberg, E. Schröder, D. C. Langreth, and B. I. Lundqvist, van der Waals Density Functional for General Geometries, Phys. Rev. Lett. 92, 246401 (2004).

[14] P. Hyldgaard, K. Berland, and E. Schröder, Interpretation of van der Waals density functionals, Phys. Rev. B 90, 075148 (2014).

[15] K. Berland, C. A. Arter, V. R. Cooper, K. Lee, B. I. Lundqvist, E. Schröder, T. Thonhauser, and P. Hyldgaard, van der Waals density functionals built upon the electron-gas tradition: Facing the challenge of competing interactions, J. Chem. Phys. 140, 18A539 (2014)

[16] J. Tao, J. Yang, and A. M. Rappe, Dynamical screening of van der Waals interactions in nanostructured solids: Sublimation of fullerenes, J. Chem. Phys. 142, 164302 (2015).

[17] P. Giannozzi et al., Quantum ESPRESSO: A modular and opensource software project for quantum simulations of materials, J. Phys.: Condens. Matter 21, 395502 (2009).

[18] M. Fuchs and X. Gonze, Accurate density functionals: Approaches using the adiabatic-connection fluctuation-dissipation theorem, Phys. Rev. B 65, 235109 (2002).

[19] E. R. Johnson and A. D. Becke, A post-Hartree-Fock model of intermolecular interactions: Inclusion of higher-order corrections, J. Chem. Phys. 124, 174104 (2006).

[20] A. Otero-de-la-Roza and E. R. Johnson, A benchmark for non-covalent interactions in solids, J. Chem. Phys. 137, 054103 (2012).

[21] J. G. Brandenburg and S. Grimme, Dispersion corrected HartreeFock and density functional theory for organic structure prediction, Top Curr. Chem. 345, 1 (2014).

[22] J. Tao and A. M. Rappe, Physical Adsorption: Theory of van der Waals Interactions between Particles and Clean Surfaces, Phys. Rev. Lett. 112, 106101 (2014).

[23] H. Peng, Z.-H. Yang, J. Sun, and J. P. Perdew, Versatile van der Waals Density Functional Based on a MetaGeneralized Gradient Approximation, Phys. Rev. X 6, 041005 (2016).

[24] T. Thonhauser, S. Zuluaga, C. A. Arter, K. Berland, E. Schröder, and P. Hyldgaard, Spin Signature of Nonlocal Correlation Binding in Metal-Organic Frameworks, Phys. Rev. Lett. 115, 136402 (2015) and also see the Supplemental Material. 
[25] J. Tao and A. M. Rappe, Accurate higher-order van der Waals coefficients between molecules from a model dynamic multipole polarizability, J. Chem. Phys. 144, 031102 (2016).

[26] J. Tao and J. P. Perdew, Non-additivity of van der Waals interactions between nanostructures, J. Chem. Phys. 141, 141101 (2014).

[27] J. Tao, Y. Mo, G. Tian, and A. Ruzsinszky, Accurate van der Waals coefficients between fullerenes and fullerene-alkali atoms and clusters: Modified single-frequency approximation, Phys. Rev. B 94, 085126 (2016).

[28] S. H. Patil and K. T. Tang, Multipolar polarizabilities and twoand three-body dispersion coefficients for alkali isoelectronic sequences, J. Chem. Phys. 106, 2298 (1997).

[29] G. K. Gueorguiev, J. M. Pacheco, and D. Tománek, Quantum Size Effects in the Polarizability of Carbon Fullerenes, Phys. Rev. Lett. 92, 215501 (2004).

[30] B. Kozinsky and N. Marzari, Static Dielectric Properties of Carbon Nanotubes from First Principles, Phys. Rev. Lett. 96, 166801 (2006).

[31] G. Y. Guo, K. C. Chu, D.-S. Wang, and C.-G. Duan, Static polarizability of carbon nanotubes: Ab initio independent-particle calculations, Comput. Mater. Sci. 30, 269 (2004).

[32] M. Ferrabone, B. Kirtman, M. Rérat, R. Orlando, and R. Dovesi, Polarizability and hyperpolarizability of BN zigzag nanotubes calculated by the coupled perturbed Kohn-Sham scheme, Phys. Rev. B 83, 235421 (2011).

[33] G. Y. Guo, S. Ishibashi, T. Tamura, and K. Terakura, Static dielectric response and Born effective charge of $\mathrm{BN}$ nanotubes from $a b$ initio finite electric field calculations, Phys. Rev. B 75, 245403 (2007).

[34] L. Wang, J. Lu, L. Lai, W. Song, M. Ni, Z. Gao, and W. N. Mei, Static and optical transverse and longitudinal screened polarizabilities of boron nitride nanotubes, J. Phys. Chem. C 111, 3285 (2007).

[35] R. Loutfy and E. Wexler, Ablative and flame-retardant properties of fullerenes, in Perspectives of Fullerene Nanotechnology, edited by E. Ōsawa (Kluwer Academic, New York, 2002), Part VII, pp. 275-280.

[36] K. Berland, Ø. Borck, and P. Hyldgaard, van der Waals density functional calculations of binding in molecular crystals, Comput. Phys. Commun. 182, 1800 (2011).

[37] M. Moalem, M Balooch, A. V. Hamza, and R. S. Ruoff, Sublimation of higher fullerenes and their interaction with silicon (100) surface, J. Phys. Chem. 99, 16736 (1995).

[38] B. Brunetti, G. Gigli, E. Giglio, V. Piacente, and P. Scardala, Some thermodynamic properties of $\mathrm{C}_{76}$ and $\mathrm{C}_{84}$, J. Phys. Chem. B 101, 10715 (1997).

[39] M. N. Magomedov, Interfullerene interaction and properties of fullerites, High Temp. (USSR) 43, 379 (2005).

[40] E. Mostaani, N. D. Drummond, and V. I. Fal'ko, Quantum Monte Carlo Calculation of the Binding Energy of Bilayer Graphene, Phys. Rev. Lett. 115, 115501 (2015).

[41] A. M. Mastral and M. S. Callén, A review on polycyclic aromatic hydrocarbon (PAH) emissions from energy generation, Environ. Sci. Technol. 34, 3051 (2000).

[42] S. D. Chakarova-Käck, A. Vojvodic, J. Kleis, P. Hyldgaard, and E. Schröder, Binding of polycyclic aromatic hydrocarbons and graphene dimers in density functional theory, New J. Phys. 12, 013017 (2010).
[43] T. Rangel, K. Berland, S. Sharifzadeh, F. Brown-Altvater, K. Lee, P. Hyldgaard, L. Kronik, and J. B. Neaton, Structural and excited-state properties of oligoacene crystals from first principles, Phys. Rev. B 93, 115206 (2016).

[44] R. Podeszwa, Interactions of graphene sheets deduced from properties of polycyclic aromatic hydrocarbons, J. Chem. Phys. 132, 044704 (2010).

[45] M. A. L. Marques, A. Castro, G. Malloci, G. Mulas, and S. Botti, Efficient calculation of van der Waals dispersion coefficients with time-dependent density functional theory in real time: Application to polycyclic aromatic hydrocarbons, J. Chem. Phys. 127, 014107 (2007).

[46] P. J. Fazen, E. E. Remsen, J. S. Beck, P. J. Carroll, A. R. McGhie, and L. G. Sneddon, Synthesis, properties, and ceramic conversion reactions of polyborazylene: A high-yield polymeric precursor to boron nitride, Chem. Mater. 7, 1942 (1995).

[47] J. R. Sanchez-Valencia, T. Dienel, O. Gröning, I. Shorubalko, A. Mueller, M. Jansen, K. Amsharov, P. Ruffieux, and R. Fasel, Controlled synthesis of single-chirality carbon nanotubes, Nature (London) 512, 61 (2014).

[48] X. Blase, A. Rubio, S. G. Louie, and M. L. Cohen, Stability and band gap constancy of boron nitride nanotubes, Europhys. Lett. 28, 335 (1994).

[49] G. Cassabois, P. Valvin, and B. Gil, Hexagonal boron nitride is an indirect bandgap semiconductor, Nat. Photon. 10, 262 (2016).

[50] G. Constantinescu, A. Kuc, and T. Heine, Stacking in Bulk and Bilayer Hexagonal Boron Nitride, Phys. Rev. Lett. 111, 036104 (2013).

[51] L. Shi, P. Rohringer, K. Suenaga, Y. Niimi, J. Kotakoski, J. C. Meyer, H. Peterlik, M. Wanko, S. Cahangirov, A. Rubio, Z. J. Lapin, L. Novotny, P. Ayala, and T. Pichler, Confined linear carbon chains as a route to bulk carbyne, Nat. Mater. 15, 634 (2016).

[52] M. Liu, V. I. Artyukhov, H. Lee, F. Xu, and B. I. Yakobson, Carbyne from first principles: Chain of $\mathrm{C}$ atoms, a nanorod or a nanorope, ACS Nano 7, 10075 (2013).

[53] O. Cretu, H.-P. Komsa, O. Lehtinen, G. Algara-Siller, U. Kaiser, K. Suenaga, and A. V. Krasheninnikov, Experimental observation of boron nitride chains, ACS Nano 8, 11950 (2014).

[54] A. Tkatchenko, R. A. DiStasio, R. Car, and M. Scheffler, Accurate and Efficient Method for Many-Body van der Waals Interactions, Phys. Rev. Lett. 108, 236402 (2012).

[55] A. A. Quong and M. R. Pederson, Density-functional-based linear and nonlinear polarizabilities of fullerene and benzene molecules, Phys. Rev. B 46, 12906(R) (1992).

[56] J. Kauczor, P. Norman, and W. A. Saidi, Non-additivity of polarizabilities and van der Waals C6 coefficients of fullerenes, J. Chem. Phys. 138, 114107 (2013).

[57] J. P. Perdew, A. Ruzsinszky, J. Sun, S. Glindmeyer, and G. I. Csonka, van der Waals interaction as a summable asymptotic series, Phys. Rev. A 86, 062714 (2012).

[58] T. Björkman, Testing several recent van der Waals density functionals for layered structures, J. Chem. Phys. 141, 074708 (2014).

[59] F. Brown-Altvater, T. Rangel, and J. B. Neaton, Ab initio phonon dispersion in crystalline naphthalene using van der Waals density functionals, Phys. Rev. B 93, 195206 (2016). 
[60] P. Erhart, P. Hyldgaard, and D. O. Lindroth, Microscopic origin of thermal conductivity reduction in disordered van der Waals solids, Chem. Mater. 27, 5511 (2015).

[61] D. O. Lindroth and P. Erhart, Thermal transport in van der Waals solids from first-principles calculations, Phys. Rev. B 94, 115205 (2016).

[62] L. Gharaee, P. Erhart, and P. Hyldgaard, Finite-temperature properties of nonmagnetic transition metals: Comparison of the performance of constraint-based semi and nonlocal functionals, Phys. Rev. B 95, 085147 (2017).

[63] K. Berland, Y. Jiao, J.-H. Lee, T. Rangel, J. B. Neaton, and P. Hyldgaard, Assessment of two hybrid van der Waals density functionals for covalent and noncovalent binding of molecules, J. Chem. Phys. 146, 234106 (2017).

[64] D. Tománek, Guide Through the Nanocarbon Jungle (Morgan \& Claypool, San Rafael, CA, 2014).
[65] W. I. F. David, R. M. Ibberson, T. J. S. Dennis, J. P. Hare, and K. Prassides, Structural phase transitions in the fullerene C60, Europhys. Lett. 18, 219 (1992).

[66] J. Kleis, E. Schröder, and P. Hyldgaard, Nature and strength of bonding in a crystal of semiconducting nanotubes: van der Waals density functional calculations and analytical results, Phys. Rev. B 77, 205422 (2008).

[67] O. A. Vydrov and T. Van Voorhis, Dispersion interactions from a local polarizability model, Phys. Rev. A 81, 062708 (2010).

[68] N. J. Silva, F. B. C. Machado, H. Lischka, and A. J. A. Aquino, Pipi stacking stacking between polyaromatic hydrocarbon sheets beyond dispersion interactions, Phys. Chem. Chem. Phys. 18, 22300 (2016).

[69] Y. Jiao, E. Schröder, and P. Hyldgaard, Signatures of van der Waals binding: A coupling-constant scaling analysis, Phys. Rev. B 97, 085115 (2018). 